\title{
Solutions to Hidden Terminal Problems in Wireless Networks
}

\author{
Chane L. Fullmer and J.J. Garcia-Luna-Aceves \\ Computer Engineering Department \\ School of Engineering \\ University of California \\ Santa Cruz, CA 95064 \\ chane,jj@cse.ucsc.edu
}

\begin{abstract}
The floor acquisition multiple access (FAMA) discipline is analyzed in networks with hidden terminals. According to FAMA, control of the channel (the floor) is assigned to at most one station in the network at any given time, and this station is guaranteed to be able to transmit one or more data packets to different destinations with no collisions. The FAMA protocols described consist of nonpersistent carrier or packet sensing, plus a collision-avoidance dialogue between a source and the intended receiver of a packet. Sufficient conditions under which these protocols provide correct floor acquisition are presented and verified for networks with hidden terminals; it is shown that FAMA protocols must use carrier sensing to support correct floor acquisition. The throughput of FAMA protocols is analyzed for single-channel networks with hidden terminals; it is shown that carrier-sensing FAMA protocols perform better than ALOHA and CSMA protocols in the presence of hidden terminals.
\end{abstract}

\section{Introduction}

The medium access control (MAC) protocol with which packetradios (or stations) can share a common broadcast channel is essential in a packet-radio network. CSMA (carrier sense multiple access) protocols [8] have been used in a number of packet-radio networks in the past [9]; these protocols attempt to prevent a station from transmitting simultaneously with other stations within its transmitting range by requiring each station to listen to the channel before transmitting. Unfortunately, "hidden terminal" problems [14] degrade the performance of CSMA substantially, because carrier sensing cannot prevent collisions in that case.

The busy tone multiple access (BTMA) protocol was the first proposal to combat the hidden-terminal problems of CSMA. BTMA is designed for station-based networks and divides the channel into a message channel and the busy-tone channel. The base sta-

\footnotetext{
This work was supported in part by the Defense Advanced Research Projects Agency under Grants DAAB07-95-C-D157 and DAAH04-96-10210.
}

tion transmits a busy-tone signal on the busy-tone channel as long as it senses carrier on the data channel. Because the base station is in line of sight of all terminals, each terminal can sense the busytone channel to determine the state of the data channel. The limitations of BTMA are the use a separate channel to convey the state of the data channel, the need for the base station to transmit the busy tone while detecting carrier in the data channel, and the difficulty of detecting the busy-tone signal in a narrowband channel.

A receiver initiated busy-tone multiple access protocol for packet-radio networks has also been proposed [17]. In this scheme, the sender transmits a request-to-send (RTS) to the receiver, before sending a data packet. When the receiver obtains a correct RTS, it transmits a busy tone in a separate channel to alert other sources nearby that they should backoff. The correct source is always notified that it can proceed with transmission of the data packet. The limitation of this scheme is that it still requires a separate busy-tone channel.

More recently, Karn [7] proposed a protocol called MACA (multiple access collision avoidance) to address the problems of hidden terminals in single-channel networks. MACA attempts to detect collisions at the receiver by establishing a request-response dialogue between senders and intended receivers. When a sending station wants to transmit, it sends a request-to-send (RTS) to the receiver, who responds with a clear-to-send (CTS) if it receives the RTS correctly. Several other MAC protocols based on similar RTS-CTS exchanges, or RTSs followed by pauses, have been proposed before and after MACA for either single-channel wireless networks or wireline local area networks $[1,4,10,11,12,16]$. The IEEE $802.11[3,6]$ committee proposed a MAC protocol for wireless LANs that includes a transmission mode based on an RTS-CTS handshake.

In this paper, we study the channel access method we have introduced previously [5] and called FAMA (floor acquisition multiple access). The objective of a FAMA protocol is for a station that has data to send to acquire control of the channel (which we call the floor) before sending any data packet, and to ensure that no data packet collides with any other packet at the receiver. To accomplish this, a FAMA protocol uses packet sensing or carrier-sensing and a three-way RTS-CTS handshake to implement what amounts to a single-channel BTMA strategy ${ }^{1}$.

Ensuring that floor acquisition is enforced among competing senders hidden from one another and who have requested the floor

\footnotetext{
${ }^{1}$ With packet sensing, stations only react to complete error free packets, and do not detect carrier on the channel. As such, they do not react to noise or partial packets in the channel.
} 
(i.e., sent an RTS) can only be achieved by the receivers. Accordingly, a FAMA protocol ensures that the CTS from a receiver lasts long enough (or is repeated enough times) to jam any hidden sender that did not hear the RTS being acknowledged. FAMA protocols constitute variations of existing MAC protocols based on RTS-CTS handshakes that eliminate collisions of data packets. Section 2 introduces two representative FAMA protocols for single-channel networks.

Although the original motivation for MACA, IEEE 802.11, MACAW [1], and BAPU [16] was to solve the hidden-terminal problems of CSMA by using RTS-CTS handshakes, it is easy to show by example that simply introducing three-way handshakes (RTS-CTS-data) or even more complex handshakes (RTS-CTSdata-ACK or others) does not suffice to eliminate all instances in which two or more senders are led to believe that they can transmit data packets to their intended receivers, only to create collisions. This is the case even if carrier sensing and RTS-CTS based handshakes are used in combination. Section 3 verifies sufficient conditions for correct floor acquisition in single-channel networks that have hidden terminals. We show that carrier sensing is necessary in FAMA protocols to eliminate hidden-terminal problems efficiently in single-channel networks. Additionally, we show that, to provide protection from hidden terminals, packet sensing has limited ability to scale or operate dynamically.

Section 4 analyzes the throughput of FAMA protocols in networks with hidden terminals. Our analysis shows that FAMA protocols that use carrier sensing attain higher throughput than FAMA protocols that use packet sensing. In the case of a network with a base station and hidden terminals, FAMA protocols achieve higher throughput than CSMA. In practice, different applications may utilize the same channel, and while some applications benefit from very large data packet sizes (e.g., transfers of video files) others do not (e.g., a telnet session). Our results show that, if data packets cannot be arbitrarily large, FAMA protocols should be used to transmit packet trains whose duration is much longer than the duration of the RTS-CTS handshake. (A packet train is made up of a bounded number of packets sent by the station holding the floor.)

Section 5 compares by simulation the performance of FAMANCS with MACAW, which is based on RTS-CTS handshake and is packet sensing [1]. Our results show very clearly that floor acquisition and carrier sensing are critical to the performance and simplicity of MAC protocols based on RTS-CTS handshakes for networks with hidden-terminals. These results, together with the results of Section 3 demonstrate that collision avoidance should be done at both sender and receiver.

\section{FAMA Protocols}

\subsection{Overview}

A FAMA protocol requires a station who wishes to send one or more packets to acquire the floor before transmitting a packet train. The floor is acquired using an RTS-CTS exchange multiplexed together with the data packets in such a way that, although multiple RTSs and CTSs may collide, data packets are always sent free of collisions. The basic principles of floor acquisition are inspired by the earlier work of Kleinrock and Tobagi on BTMA [14] and the provision of priority acknowledgments in ALOHA and CSMA [15].
To acquire the floor, a station sends an RTS using either packet sensing or carrier sensing. The first variant corresponds to using the ALOHA protocol for the transmission of RTSs; the second consists of using a CSMA protocol to transmit RTSs. A station sends a CTS after receiving an error-free RTS addressed to it. When a station receives an error-free CTS, it knows that the floor has been acquired by the station to whom the CTS is addressed. After floor acquisition, either the floor holder or any of the receivers addressed by the floor holder are able to send data packets and acknowledgments free of collisions over the channel. Any reliable link control scheme can be implemented on top of FAMA between the floor holder and the stations with whom it wishes to communicate. This is accomplished by forcing stations that do not have the floor to wait a predefined minimum amount of time (at least twice the maximum propagation delay) before being able to bid for the floor. This is similar to the schemes for the provision of priority acknowledgments proposed for CSMA and ALOHA by Kleinrock and Tobagi [15].

To ensure that floor acquisition is enforced among competing senders hidden from one another and who have requested the floor (i.e., sent an RTS), the CTS sent by a receiver is guaranteed to last long enough (or to be repeated enough times) to jam any hidden sender that did not hear the RTS being acknowledged. This corresponds to a single-channel BTMA scheme in which sensing of error-free CTSs (for packet sensing) or the carrier of a CTS (for carrier sensing) over the same data channel is used instead of a busy-tone signal sent over a separate channel.

When a station with data to send fails to acquire the floor or detects the floor being held by another station, it must reschedule its bid for the floor. This can be done using different persistence and backoff strategies. In this paper, we consider only non-persistent protocols. We also specify FAMA protocols that use a uniform distribution when choosing backoff times; however, other backoff strategies can be adopted (e.g., see those proposed for MACAW [1]).

To simplify our analysis and description of FAMA protocols, we do not address the effect of acknowledgments in the rest of this paper, and assume the simplest three-way handshake (RTS-CTSdata) with no acknowledgments sent after data packets.

\subsection{FAMA-NCS}

The first variant of FAMA, which we call FAMA-NCS (for nonpersistent carrier sensing) combines non-persistent carrier sensing with the RTS-CTS exchange. This variant of FAMA is similar to the protocol proposed for IEEE 802.11 [2], and Apple's Local Talk Link Access protocol [12]. However, those and other protocols based on carrier sensing and RTS-CTS handshakes do not guarantee floor acquisition in networks with hidden terminals.

The length of an RTS is larger than the maximum channel propagation delay plus any processing time. This is required to avoid one station hearing a complete RTS before another has started to receive it.

The length of a CTS in FAMA-NCS is larger than the aggregate of the length of an RTS plus one maximum round trip time across the channel, the transmit to receive turn around time, and any processing time. The relationship of the size of the CTS to the RTS gives the CTS dominance over the RTS in the channel. Once a station has begun transmission of a CTS, any other station within range of it that transmits an RTS simultaneously (i.e., within one propagation delay of the beginning of the CTS) will hear at least a 
portion of the dominating CTS after returning from transmit mode and backoff, thereby allowing the data packet that will follow to arrive free from collision. The dominating CTS plays the role of a busy tone by providing a jamming signal to possible interfering transmitters within range of the sender of the CTS.

Figure 1 shows an example of how the CTS dominance operates in more detail. Station $B$ is sending a CTS while station $A$ is attempting to send its RTS and acquire the floor. $A$ can send its RTS no later than $\tau$ seconds after $B$ starts its CTS (otherwise it would hear the CTS and wait). In this example $B$ 's CTS arrives at $A$ just as $A$ begins its RTS transmission (Figure 1a). Because $B$ 's CTS is longer than the RTS plus the transmit to receive turnaround time, $A$ hears the overlap as noise and backs off. On the other hand, $A$ can begin its RTS and interfere with $B$ 's CTS no earlier than $\tau$ seconds before $B$ begins its CTS transmission (otherwise $B$ would have detected the signal and not sent the CTS). In this case (shown in Figure $1 \mathrm{~b}$ ), the CTS arrives at $A 2 \tau$ seconds after that of $B$ 's RTS. Again, because the CTS is longer than the RTS plus the transmit to receive turnaround time, $A$ hears the end of the CTS as noise and backs off.

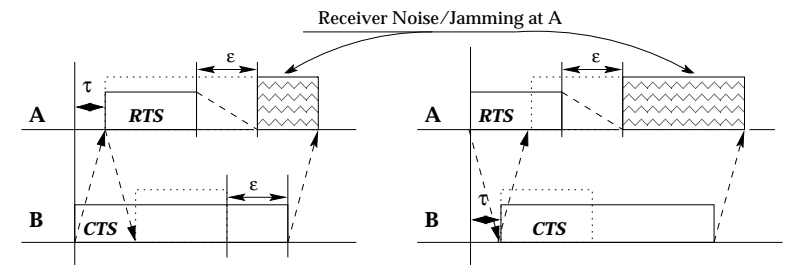

a) A sends RTS after B's CTS

b) A sends RTS before CTS at B

Figure 1:

Dominance of the CTS in FAMA for hidden-terminal:

a) $A$ begins its RTS just as $B$ 's CTS arrives at $A$

b) $A$ begins its RTS $\tau$ seconds in advance of the CTS from $B$

Figure 2 specifies FAMA-NCS in detail. A station that has just been initialized must wait the time it takes to transmit the maximum-size data packet in the channel plus one maximum round-trip time across the channel. This allows any neighboring station involved in the process of receiving a data packet to complete the reception un-obstructed. The initialization time also gives the station the ability to learn of any local traffic in progress. If no carrier is detected during the initialization period, the station transitions to the PASSIVE state. Otherwise, it transitions to the REMOTE state. A station can only be in the PASSIVE state if it is properly initialized (i.e., has no packet to send, and senses an idle channel). In all other states, the station must have listened to the channel for a time period that is sufficiently long for any neighbor involved in receiving data to have finished.

A station that is in the PASSIVE state and senses carrier transitions to the REMOTE state. On the other hand, a station that receives a packet to send in the PASSIVE state transmits an RTS and transitions to the RTS state. The sending station waits long enough for the destination to send the CTS. If the CTS is not received within the time allowed, the sender transitions to the BACKOFF state. If the sender hears noise on the channel after its RTS, it assumes a collision with a neighbor's dominating CTS and waits long enough for a maximum-length data packet to be received. Otherwise, upon receiving the CTS, the sender transmits its data packet. Because the
CTS could be corrupted at the sender, once the destination station sends its CTS, it only needs to wait one maximum round-trip time to sense the beginning of the data packet from the source. If the data packet does not begin, the destination transitions either to the BACKOFF state (if it has traffic pending) or to the PASSIVE state.

In the BACKOFF state, if no carrier is detected during the entire backoff waiting period computed by the station, the station transmits an RTS and transitions to the RTS state as before; otherwise, after sensing carrier the station transitions to the REMOTE state.

For stations in the REMOTE state, FAMA-NCS enforces different waiting periods on passive stations (those stations not directly involved in the current transmission period) based on what was last heard on the channel. Any passive station that detects carrier transitions to the REMOTE state, and after the channel clears the waiting period is determined as follows:

- After hearing an RTS for another station, the station must wait long enough for a CTS to be transmitted by the destination and received by the sender, and the data packet to begin.

- After hearing a CTS from another station, the station must wait long enough to allow the other station to receive its data packet.

- After hearing a data packet, the waiting time is the enforced FAMA waiting period.

- After hearing noise (colliding control packets) on the channel, the waiting period must be long enough to allow another station time to receive a maximum size data packet.

The channel becomes idle when all stations are in either the PASSIVE or BACKOFF state. The next access to the channel is driven by the arrival of new packets to the network and retransmission of packets that have been backed off.

To increase the efficiency of the channel, a station that has successfully acquired the floor can dynamically send multiple packets together in a train, bounded by an upper limit. To allow this to be successful in a hidden-terminal environment, the destination station must alert its neighbors that it has more data packets coming, and to continue to defer their transmissions. FAMA-NCS uses a simple handshake mechanism to support packet trains.

Because a receiver's neighbors are only required to defer transmission for the length of a maximum-sized data packet, data packets are not concatenated. Instead, a CTS is sent after each data packet in a packet train (except the last packet in the train).

If the sending station has multiple packets to send, it sets a MORE flag in the header of the data packet. When the destination receives the data packet and sees the MORE flag set, it immediately responds with a CTS, just as when hearing an RTS. This CTS alerts all neighbors that might interfere with the next data packet that they must continue to defer.

Additionally, stations in the REMOTE state must extend their waiting period after hearing a data packet with the MORE flag set to allow additional time for the sender to receive the CTS from the destination signaling that it can receive the next data packet.

\subsection{FAMA-NPS}

The second variant of FAMA that we address is called FAMA-NPS (for non-persistent packet sensing). The key aspect of this variant of FAMA protocols is that stations do not sense the channel before 

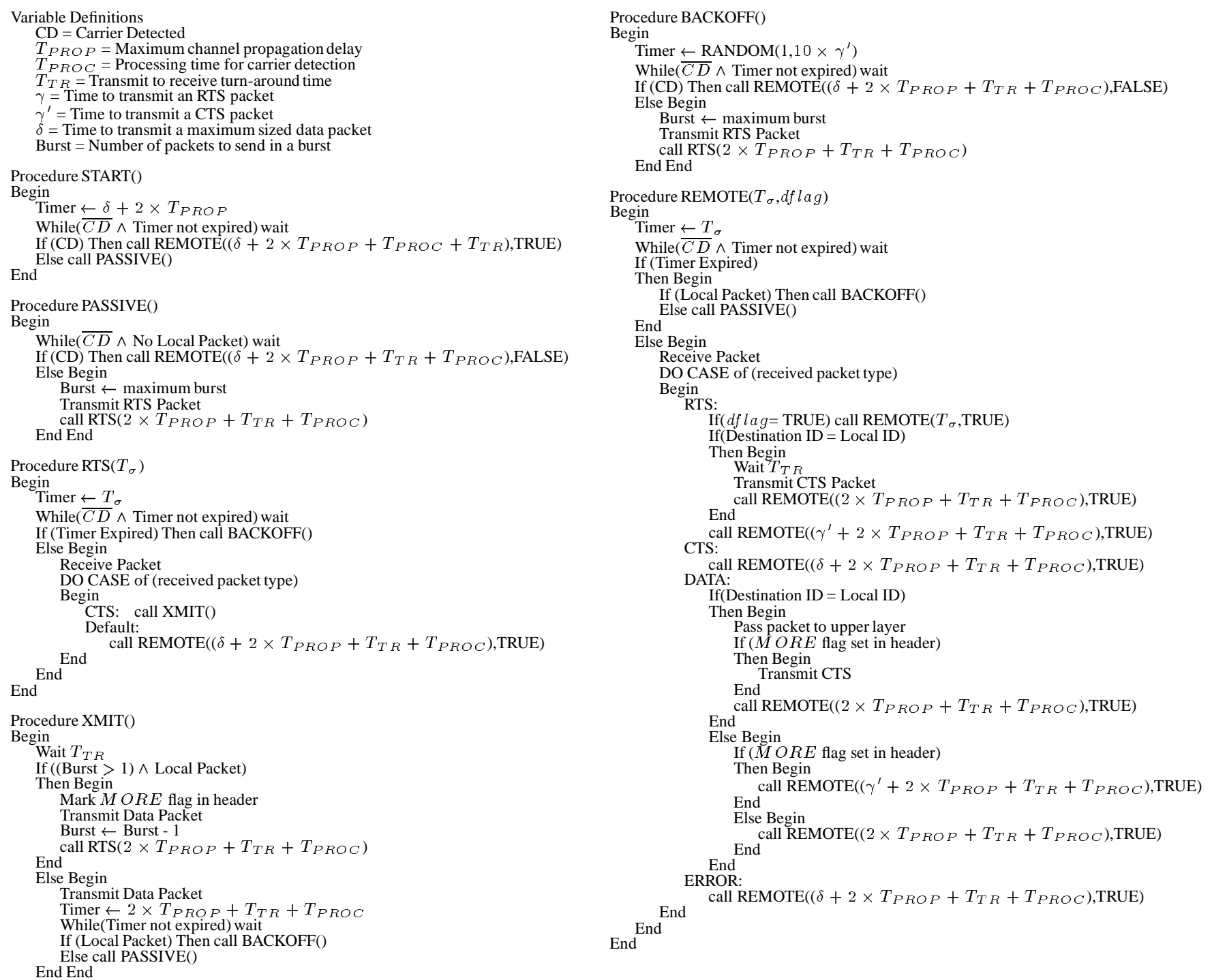

Figure 2: FAMA-NCS Specification

transmissions. It basically consists of the MACA protocol (Multiple Access Collision Avoidance) recently proposed by Karn [7]. Fig. 3 specifies FAMA-NPS in detail.

Section 3 shows that, for a FAMA protocol with packet sensing to work with hidden terminals, the CTSs must be transmitted multiple times, which means that floor acquisition can be supported efficiently only in fully connected networks. Accordingly, our specification of FAMA-NPS assumes that it is used in a fully connected network and that a CTS is transmitted only once. RTSs and CTSs have the same duration, which is longer than one maximum roundtrip delay.

A station that has a data packet to send and that is not expecting to hear a CTS or a data packet first transmits an RTS to the receiver. When a station processes a correct RTS, it defers transmission of any RTS for an amount of time specified in the RTS. If the RTS is addressed to the station, it sends a CTS and waits long enough for an entire data packet to arrive from the sender. Following the deferment specified by the RTS, a station with a packet to send waits a random waiting period before it transmits an RTS.

MACA and improvements over it are also discussed in detail by
Bharghavan et al. [1].

\section{Correct Floor Acquisition}

\subsection{Carrier-Sensing Protocols}

For a FAMA protocol to provide correct floor acquisition, it must ensure that each new packet, or any of its retransmissions, is sent to the channel within a finite time after it becomes ready for transmission, and that a data packet does not collide with any other transmission.

Theorem 1 below shows that FAMA-NCS provides correct floor acquisition if an RTS lasts longer than the maximum propagation delay and a CTS lasts longer than the time it takes to transmit an RTS, plus a maximum round-trip time and a maximum hardware transmit-to-receive transition time. This takes care of the case in which the transition times are shorter than propagation delays; in most cases however, the transmit-to-receive transition times are much larger than the propagation delays. We make the following 

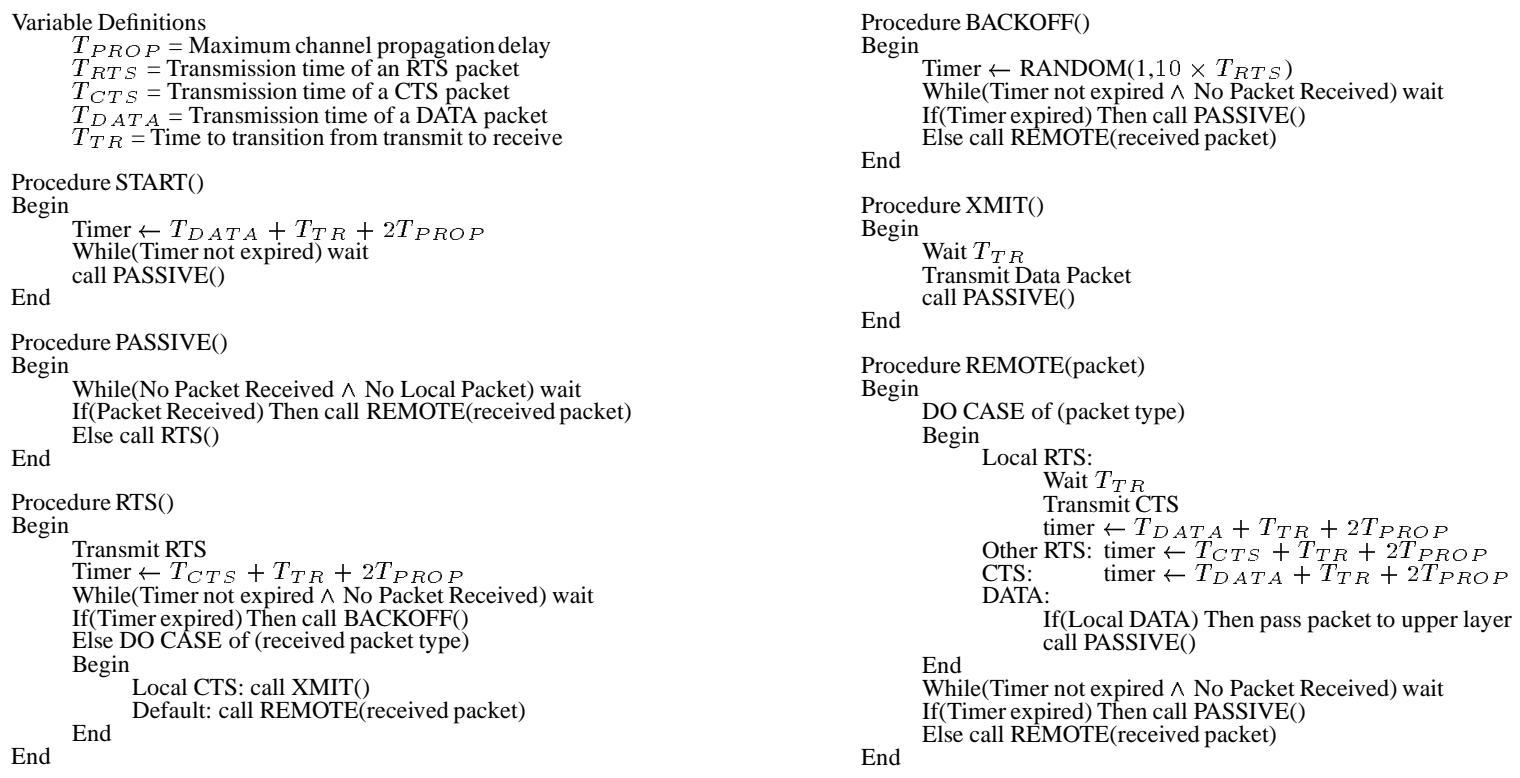

Figure 3: FAMA-NPS Specification

assumptions to prove the theorem: ${ }^{2}$

A0) The maximum end-to-end propagation time in the channel is $\tau<\infty$.

A1) A packet sent over the channel that does not collide with other transmissions is delivered error free with probability $p>0$.

A2) A station sends an RTS to the intended destination and receives a CTS in return that does not collide with any other transmission with probability larger than 0 .

A3) All stations execute FAMA-NCS correctly.

A4) The transmission time of an RTS is $\gamma<\infty$, the transmission time of a CTS is $\gamma^{\prime}<\infty$, the maximum transmission time of a data packet is $\delta<\infty$, and the hardware transmit-to-receive transition time is $2 \tau<\varepsilon<\infty$.

A5) There is no capture or fading on the channel.

A6) Any overlap by transmissions at a particular receiver causes that receiver to not understand either packet.

Theorem 1 FAMA-NCS provides correct floor acquisition in the presence of hidden terminals, provided that $\gamma>\tau$ and $\gamma+2 \tau+\varepsilon<$ $\gamma^{\prime}<\infty$.

Proof: Figure 4 illustrates any possible case of hidden terminals with respect to a given pair of source $S$ and receiver $R$. Station $L$ characterizes any neighbor of $S$ that is hidden from $R$ but can cause interference at $S$. Station $K$ characterizes any neighbor of $L$ hidden from $S$ that can cause interference at $L$ and can prevent $L$ from following $S$ 's dialogue with $R$. Similarly, Station $X$ is a neighbor of $R$ that is hidden from $S$ but can cause interference at $R$; and station $Y$ is a neighbor of $X$ that is hidden from $R$ and can prevent $X$ from following $R$ 's dialogue with $S$. The proof must show that, if $S$ sends a data packet to $R$, no other transmission

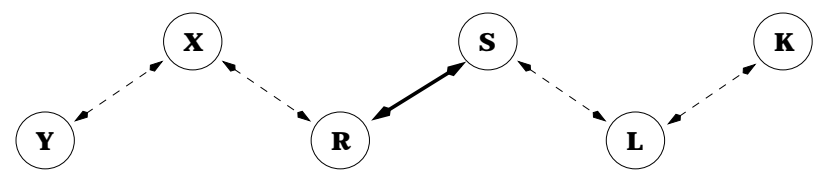

Figure 4: Stations involved in interference of the exchange between $S$ and $R$

can collide with it, regardless of the possible transmissions of other interfering nodes.

For $S$ to be able to send data packets to $R$, it must first receive a CTS from $R$. Without loss of generality, assume that, at time $t_{0}, S$ sends an RTS to $R$.

Because the channel has a minimum propagation delay larger than 0 , any neighbor of $S$ (e.g., Station $L$ ) must start receiving $S$ 's RTS at time $t_{0}^{L}>t_{0}$. If $L$ receives $S$ 's RTS correctly, then it must back off for a period of time larger than $2 \tau+\gamma^{\prime}$ after the end of $S$ 's RTS reaches $L$, which means that $L$ backs off for $\gamma+2 \tau+\gamma^{\prime}$ seconds after $t_{0}^{L}$. Alternatively, if the RTS reaches $L$ in error or Station $K$ 's transmission interferes with $S$ 's RTS at Station $L$, then, starting with the end of carrier, Station $L$ must back off for a period of time larger than $2 \tau+\delta$. The minimum amount of time that $L$ must back off then corresponds to the case in which the end of carrier coincides with the end of $S$ 's RTS. Accordingly, $L$ must back off for $\gamma+2 \tau+\delta$ seconds after $t_{0}^{L}$. It follows that the RTS sent by $S$ at time $t_{0}$ forces any neighbor of $S$ other than $R$ to back off until time $t_{1}>t_{0}+\gamma+\gamma^{\prime}+2 \tau$.

If the RTS is received at Station $R$ with errors or collides with transmissions from other neighbors of $R$ who are hidden from $S$ (e.g., $X$ ), then $R$ cannot send a CTS and $S$ cannot send its data packet in return.

Assume that $S$ 's RTS is received correctly by $R$ at time $t_{2}$. If $S$ receives $R$ 's CTS with errors or the CTS collides with transmissions from neighbors of $S$ hidden from $R$ (e.g., $L$ ), then $S$ cannot

\footnotetext{
${ }^{2}$ Similar results can be obtained under different assumptions using a similar approach to the one presented here.
} 
send its data packet.

For the rest of the proof, assume that the RTS that $S$ sends at time $t_{0}$ is received error free at station $R$ within one maximum propagation delay, which means that $R$ must start sending its CTS to $S$ at time $t_{2} \leq t_{0}+\gamma+\tau$ (given that zero processing delays are assumed). This CTS must reach $S$ within one maximum propagation delay after $R$ sends it. Therefore, $S$ must receive $R$ 's entire CTS at time $t_{3} \leq t_{2}+\gamma^{\prime}+\tau=t_{0}+\gamma+\gamma^{\prime}+2 \tau$.

Because $t_{1}>t_{3}$, it follows that any potential interfering neighbor of $S$ (e.g., $L$ ), must back off long enough for $S$ to be able to receive $R$ 's CTS without collisions.

Station $S$ must start to receive $R$ 's CTS no later than $\tau$ seconds after $R$ starts its transmission, and must receive $R$ 's entire CTS and send its data packet at time $t_{4} \leq t_{2}+\tau+\gamma^{\prime}$. In turn, Station $R$ must receive the end of $S$ 's data packet by time $t_{5} \leq t_{4}+\delta+\tau \leq$ $t_{2}+2 \tau+\gamma^{\prime}+\delta$.

On the other hand, any station $X$ other than $S$ within range of $R$ must start receiving $R$ 's CTS at time $t_{2}^{X}>t_{2}$. If $X$ receives $R$ 's CTS with no errors, then it must back off for a period of time larger than $2 \tau+\delta$ after the end of $R$ 's CTS reaches $X$, which means that $X$ backs off for $2 \tau+\delta+\gamma^{\prime}$ seconds after $t_{2}^{X}$. Conversely, if $R$ 's CTS reaches $X$ in error or a transmission from one of its neighbors hidden from $R$, call it $Y$, interferes with the CTS, then, starting with the end of carrier, $X$ must back off for more than $\delta+2 \tau$ seconds. The minimum amount of time that $X$ backs off corresponds to the case in which the time when $X$ detects the end of carrier equals the time when $X$ receives $R$ 's entire CTS; therefore, $X$ must back off for $2 \tau+\delta+\gamma^{\prime}$ seconds after $t_{2}^{X}$. It follows that the CTS sent by $R$ at time $t_{2}$ forces $X$ and any neighbor of $R$ other than $S$ to back off until time $t_{6}>t_{2}+2 \tau+\gamma^{\prime}+\delta$.

Because $t_{6}>t_{5}$, it follows that Station $X$ and any other potential interfering neighbor of $R$ must back off long enough for $R$ to be able to receive $S$ 's data packet without collisions. Accordingly, it is true that FAMA-NCS allows a station to transmit a data packet only after a successful RTS-CTS exchange and no data packet collides with other transmissions.

\subsection{Packet-Sensing Protocols}

The following theorem shows that, although a FAMA protocol based on packet sensing can support correct floor acquisition in the presence of hidden terminals, it would be impractical to do so in a dense network because CTSs must be repeated too many times. The theorem relies on the following assumptions, which extend or modify the assumptions introduced in Section 3.1:

A7) A station only recognizes complete packets, and cannot understand noise, or partial packets.

A8) $N$ is the total number of neighbors any node in the network may have, plus the maximum number of neighbors any one of those neighbors may have (not including the sender and intended receiver).

A9) $\gamma$ is the size of an RTS and CTS.

To understand the problem, assume that Station $S$ sends an RTS that is received correctly at Station $R$, then $R$ immediately begins transmission of a CTS to $S$. Figures 5 and 6 show two cases where the CTSs are not understood by stations in $R$ 's neighborhood. In the first case, station $X_{a}$ in $R$ s neighborhood transmits an RTS to
$R$, blocking itself and all other stations in $R$ s neighborhood from understanding the first and second CTSs. In the second case, a station in the neighborhood of $X_{a}$ (and not $R$ or $S$ ) transmits an RTS that blocks $R$ s CTS from $X_{a}$ allowing $X_{a}$ to transmit an RTS itself blocking additional CTSs. In either case, at least $X_{a}$ does not understand the CTS and can transmit an RTS that collides at $R$ with the data packet from $S$ if not enough CTSs are sent by station $R$.
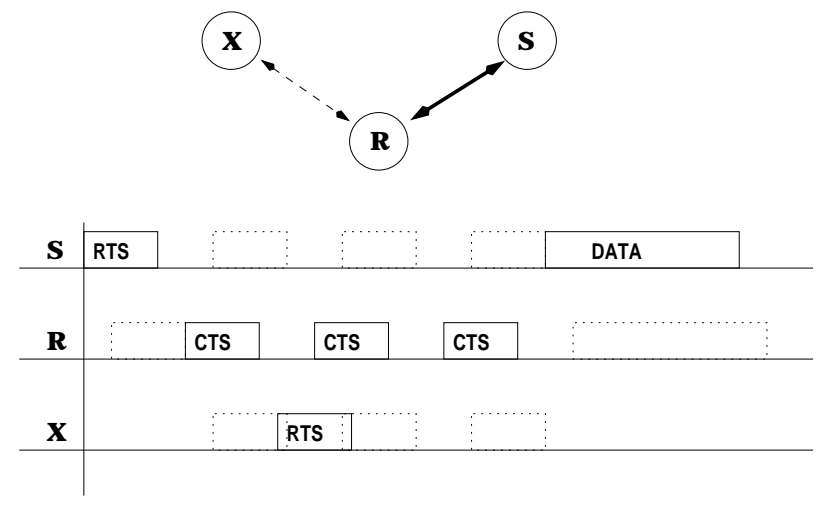

Case 1

Figure 5: Packet Sensing with hidden terminals, $\mathrm{N}=1$

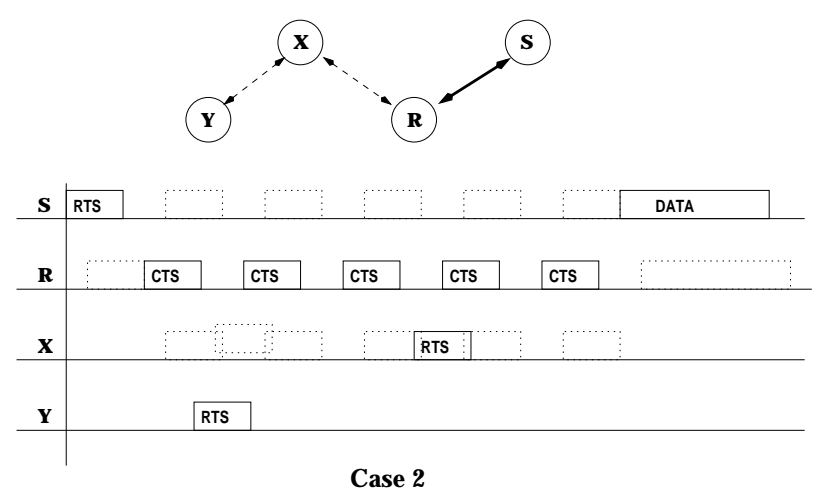

Figure 6: Packet Sensing with hidden terminals, $\mathrm{N}=2$

To resolve the contention in the first case, the receiver needs to send at least three separate CTSs $(N=1)$. This is necessary, because a station considers the channel clear until any packet transmission is completely received free of error, and until that point there is no detection of traffic on the channel and transmissions are possible. As such, station $X_{a}$ can transmit its RTS just before the very end of receiving the CTS from $R$, and in the process also transmits over the beginning of the next CTS. $X_{a}$ waits to get the CTS for it from $R$ and instead sees the CTS to $S$, and defers further transmission.

In the second case, $R$ must send at least five CTSs $(N=2)$. Here, the neighbor of $X_{a}$ transmits an RTS that can collide with the first and second CTS blocking them from $X_{a}$, allowing it to send an RTS masking the third and fourth CTSs. The fifth CTS will be understood at $X_{a}$ forcing it to defer after that point.

Theorem 2 A FAMA protocol using packet sensing provides correct floor acquisition in the presence of hidden terminals, provided that the receiver transmits at least $(2 N+1)$ CTSs in response to an 
RTS and the minimum waiting time required after an unsuccessful RTS is greater than $2 N(\gamma+2 \tau)$.

Proof:

It is possible that each station in $R$ 's neighborhood, and their neighbors, can interact sending RTSs in such a way that at least one of $R$ 's neighbors has $2 N$ consecutive CTSs blocked (two for each RTS sent), where $N$ is the total number stations in $R$ 's neighborhood, plus the maximum number of stations in any of these station's own neighborhood and hidden to $R$. As such, $R$ needs to transmit $2 N+1$ CTSs to guarantee that all of its neighbors understand at least one of the CTSs, and defer any further transmissions. Figure 7 shows an example in which $R$ has $n$ neighbors and neighbor $X_{a}$ has the maximum number of neighbors, $m$, that cannot hear station $R$. In this example $N=n+m$ and station $R$ would have to transmit a minimum of $2 \times(n+m)+1$ CTSs to ensure all of it's neighbors were in a deferred state, and allow the data packet from $S$ to be delivered collision free.

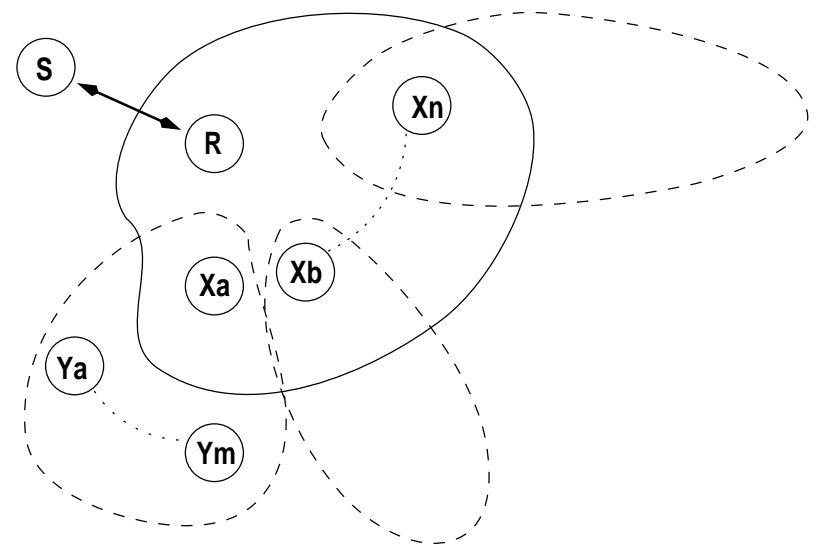

Figure 7: NPS Neighborhoods with hidden terminals

A station $X_{a}$ that sends an RTS must wait to understand the CTS from its intended destination. Otherwise, if no response is heard from its destination, it must wait a long enough time to allow another (neighboring) receiver $R$ to send its CTSs. A receiver must send at least $2 N+1$ consecutive CTSs to ensure station $X_{a}$ understands at least one (possibly the last) CTS. If $X_{a}$ 's RTS is the first to start blocking the CTSs, it may have to wait for another $2 N-1$ blocked CTSs before understanding a CTS. With a $2 \tau$ space between CTSs, the maximum time after sending an unsuccessful RTS station $X_{a}$ can determine the channel is again clear is $2 N(\gamma+2 \tau)$ seconds. Because station $X_{a}$ does not ever know in advance that it is, or not, blocked by the first set of CTSs it must always assume so and wait a minimum of $2 N(\gamma+2 \tau)$ seconds to transmit again if no neighbor's CTS is understood before then.

It is easy to see that, as the size of the network increases, any receiver $R$ must send at least $2 N+1$ CTSs to ensure that its neighbors are aware of its pending reception of a data packet.

Use of multiple channels with a common control channel has been suggested to solve the hidden terminal problem for packet sensing protocols [16]. However, collision of data packets can still occur. For instance, assume there are two channels, one for control and one for data in the network shown in Fig. 4. Station $S$ transmits an RTS to station $R$ at time $t_{0}$. Station $R$ responds with a CTS at time $t_{1}$, additionally station $X$ transmits an RTS to station
$Y$ at time $t_{1}$, masking the CTS from station $R$. At time $t_{2} S$ begins transmitting the data packet, and $Y$ sends the CTS to $X$. Station $X$ receives the CTS in the clear and begins its data packet at time $t_{3}$, which collides with the data packet from $S$ at $R$. Therefore, even with multiple channels, packet sensing cannot be guaranteed to eliminate collisions of data packets.

\section{Comparative Throughput Analysis}

\subsection{Assumptions and Notations}

As we have shown in Section 3, carrier sensing is needed to attain correct floor acquisition without sacrificing performance, which makes FAMA-NCS the only practical solution to the hiddenterminal problem. Therefore, the rest of this section concentrates on FAMA-NCS only.

We present an approximate throughput analysis of FAMA-NCS that assumes the same traffic model first introduced for CSMA [8] to analyze the throughput of CSMA protocols, and the conditions for floor acquisition derived in Section 3. The throughput of nonpersistent CSMA used to compare with FAMA-NCS's was reported by Kleinrock and Tobagi [8].

There is an infinite number of stations who constitute a Poisson source with an aggregate mean generation rate of $\lambda$ RTS packets per unit time. Any station can listen to the transmissions of any other station.

Each station is assumed to have at most one data block to send at any time. In all protocols, a station transmits the entire data block as a single packet (which is the case of CSMA and MACA as it is described in [7]) or as multiple contiguous packets (which is the case of FAMA-NCS). The size of a data block is assumed to be $\delta$ seconds. RTSs last $\gamma$ seconds, CTSs last $\gamma^{\prime}$ seconds, and the maximum end-to-end propagation delay of the channel is $\tau$ seconds. Collisions (e.g., RTS packets in FAMA-NCS, data packets in CSMA) can occur in the channel, and we assume that, when a station has to retransmit a packet, it does so after a random retransmission delay that is much larger than $\delta$ on the average. The average channel utilization is given by [8]

$$
S=\bar{U} /(\bar{B}+\bar{I})
$$

where $\bar{B}$ is the expected duration of a busy period, defined to be a period of time during which the channel is being utilized; $\bar{I}$ is the expected duration of an idle period, defined as the time interval between two consecutive busy periods; and $\bar{U}$ is the average time during a busy period that the channel is used for transmitting user data successfully.

The channel is assumed to introduce no errors, so packet collisions are the only source of errors, and stations detect such collisions perfectly. To further simplify the problem, we assume that two or more transmissions that overlap in time in the channel must all be retransmitted, and that a packet propagates to all stations in exactly $\tau$ seconds [8]. The later assumption provides a lower bound on the performance of the protocols we analyze.

Of course, this model is only a rough approximation of the real case, in which a finite number of stations access the same channel, stations can queue multiple packets for transmission, and the stations' transmissions and retransmissions (of RTS or data packets) are correlated (e.g., a failed RTS is followed by another RTS within a bounded time). However, this model is a simple tool that 
helps us to understand why it is beneficial to listen for any type of channel activity, rather than for specific packet types, and provides additional insight on the performance of FAMA protocols and the impact of channel speed and propagation delay on the floor acquisition technique.

\subsection{FAMA-NCS Throughput}

To study the performance of FAMA-NCS under hidden-terminals, we adopt the same tractable model first used by Tobagi and Kleinrock [14] to analyze the impact of hidden terminals in CSMA. The model includes the same assumptions made in Section 4.1, and a system configuration consisting of a large number of terminals communicating with a single base station over a single channel. All terminals are within line-of-sight and range of the base station, but they may be hidden from one another. The population of terminals is partitioned into $N$ independent groups [14], such that all terminals within the same group can hear one another and the base station, and any two terminals from different groups are hidden from each other. Each group $i$ consists of a large number of terminals who collectively form an independent Poisson source with an aggregate mean rate of $\lambda_{i}$ floor requests per second, such that $\Sigma_{i}^{N} \lambda_{i}=\lambda$.

The result proved in Theorem 3 helps in predicting the degradation in throughput in FAMA-NCS due to hidden terminals. Our simulation results in Section 5 confirm our analysis for both multihop networks and networks with base stations and hidden nodes.

Theorem 3 The throughput of FAMA-NCS for a system with $N$ independent groups of hidden terminals is given by Eq. (2).

Proof: Consider the time line for the base station; it consists of a sequence of busy and idle periods. Because FAMA-NCS provides correct floor acquisition, collisions can occur only among RTSs. Therefore, because no successful RTS can overlap at all with any other RTS and because a successful transmission period is detected by all groups and forces an idle time of $2 \tau$ seconds, a busy period consists of either a single failed transmission period or a single successful transmission period.

An RTS originated from any node $s$ in Group $i$ is successful if no other RTS from any group collides with $s$ 's RTS. Within Group $i$, the vulnerability period of $s$ 's RTS is $\tau$ seconds, because all nodes in Group $i$ can detect carrier $\tau$ seconds after the beginning of the RTS. Accordingly, an RTS is successful within its own Group $i$ with probability $e^{-\lambda_{i} \tau}$. In contrast, the vulnerability period of an RTS with respect to other groups is $2 \gamma$, because nodes in hidden groups cannot hear $s$ 's transmissions. Accordingly, an RTS is successful with respect to a Group $j$ other than its own with probability $e^{-\lambda_{j}(2 \gamma)}$. Because all groups are independent, it follows that an RTS from Group $i$ is successful at the base station with the following probability:

$$
P_{S_{i}}=e^{-\lambda_{i} \tau} \prod_{j \neq i}^{N} e^{-\lambda_{j}(2 \gamma)}
$$

Therefore, the probability that an RTS from any one group is successful equals

$$
P_{S}=\frac{1}{N} \sum_{i=1}^{N}\left(e^{-\lambda_{i} \tau} \prod_{j \neq i}^{N} e^{-\lambda_{j}(2 \gamma)}\right)
$$

A successful transmission period in the time line of the base station consists of an RTS preceded by a propagation delay from the sender, a CTS and propagation delay back to the sender, another propagation delay for the data packet to reach the base station followed by the data packet. Accordingly, the time for a successful transmission, $T$, is

$$
T=\gamma+\gamma^{\prime}+3 \tau+\delta
$$

There are two types of failed transmission periods. If only one of the groups sends RTSs in a transmission period, its average duration in the time line of the base station equals $T_{F_{1}}=\gamma+\bar{Y}$, where $\bar{Y}$ is the same as in the fully-connected network case. Noting that $\bar{Y} \leq \tau$, we use the following bound for simplicity:

$$
T_{F_{1}} \leq \gamma+\tau
$$

The probability that an RTS from any given group is successful with respect to the rest of the other groups is given by

$$
P_{S E}=\frac{1}{N} \sum_{i=1}^{N}\left(\prod_{j \neq i}^{N} e^{-\lambda_{j}(2 \gamma)}\right)
$$

If more than one group sends RTSs in a failed transmission period, the failed transmission period consists of multiple overlapping transmission periods with average durations of $T_{F_{1}}$ seconds. Because groups are hidden and independent from each other, the length of the average failed transmission period in this case can be obtained by treating this case as an ALOHA channel with $N$ stations, in which a station $i$ corresponds to Group $i$ and has an aggregate rate of $\lambda_{i}$. An average failed transmission period consists of a geometrically-distributed indefinite number $(L)$ of interarrival times whose average duration is $\bar{f}$ seconds (the average time between failed arrivals), plus the duration of an RTS $(\gamma)$. The values for $\bar{L}$ and $\bar{f}$ are derived in [13] for pure ALOHA as functions of $\lambda$ and, according to our notation, $\delta$. Substituting $\gamma$ for $\delta$ in such results we obtain $e^{\lambda \gamma}$ and $(\lambda \gamma)^{-1}-e^{-\lambda \gamma} /\left(1-e^{-\lambda \gamma}\right)$, respectively. Therefore, when the first RTS of the transmission period collides with other RTSs, the average time of a failed transmission period, $T_{F R T S}$, equals

$$
T_{F R T S}=\left[\frac{e^{\lambda \gamma}-1-\lambda \gamma}{\lambda \gamma\left(1-e^{-\lambda \gamma}\right)}\right]+\gamma
$$

To make use of prior results, we make the simplifying assumption that $N$ is very large. Accordingly, we approximate the average duration of the failed transmission period by substituting the upper bound of Eq. (6) for $\gamma$ in Eq. (8), which yields

$$
T_{F_{2}}=\left[\frac{e^{\lambda(\gamma+\tau)}-1-\lambda(\gamma+\tau)}{\lambda(\gamma+\tau)\left(1-e^{-\lambda(\gamma+\tau)}\right)}\right]+(\gamma+\tau)
$$

Accordingly, the average busy period lasts

$\bar{B}=P_{S E}\left(e^{-\lambda_{i} \tau}(T)+\left(1-e^{-\lambda_{i} \tau}\right)\left(T_{F_{1}}\right)\right)+\left(1-P_{S E}\right)\left(T_{F_{2}}\right)$

Substituting Eqs. (7), (6) and (9) in the above Eq. (10), we obtain Eq. (11).

The average idle period lasts $2 \tau$ seconds after every successful data packet transmission plus an average interarrival time of RTSs from all groups; therefore, we have

$$
\bar{I}=\frac{1}{\lambda}+2 \tau \cdot P_{S}
$$




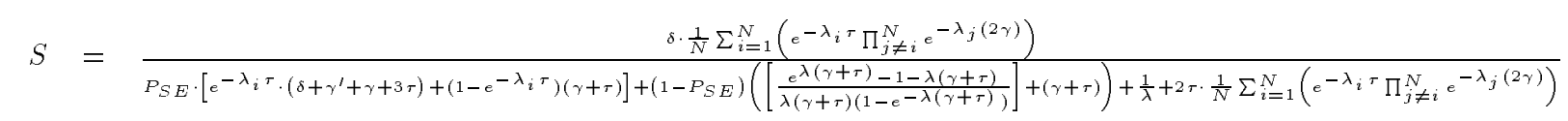

$$
\begin{aligned}
& \text { where } P_{S E}=\frac{1}{N} \sum_{i=1}^{N}\left(\prod_{j \neq i}^{N} e^{-\lambda_{j}(2 \gamma)}\right)
\end{aligned}
$$

$$
\bar{B}=\frac{1}{N} \sum_{i=1}^{N}\left(\Pi_{j \neq i}^{N} e^{-\lambda_{j}(2 \gamma)}\right)\left(e^{-\lambda_{i} \tau}\left(\delta+\gamma^{\prime}+\gamma+3 \tau\right)\left(1-e^{-\lambda_{i} \tau}\right)(\gamma+\tau)\right)+\left(1-\frac{1}{N} \sum_{i=1}^{N}\left(\Pi_{j \neq i}^{N} e^{-\lambda_{j}(2 \gamma)}\right)\right)\left(\left[\frac{e^{\lambda(\gamma+\tau)}-1-\lambda(\gamma+\tau)}{\lambda(\gamma+\tau)\left(1-e^{-\lambda(\gamma+\tau)}\right)}\right]+(\gamma+\tau)\right)
$$

The average utilization time is simply the proportion of time in which useful data are sent during a successful busy period, and

$$
\bar{U}=\delta \cdot P_{S}
$$

Substituting Eqs. (11), (12), and (13) into Eq. (1), we obtain the desired result. $\square$

In the limit, as $N \rightarrow \infty$, we obtain that the average throughput in any given system becomes

$$
S=\frac{\delta}{\delta+\gamma^{\prime}+\gamma+5 \tau+\left(e^{2 \lambda \gamma}-1\right)\left(\left[\frac{e^{\lambda(\gamma+\tau)}-1-\lambda(\gamma+\tau)}{\lambda(\gamma+\tau)\left(1-e^{-\lambda(\gamma+\tau)}\right)}\right]+\gamma+\tau\right)+\frac{e^{2 \lambda \gamma}}{\lambda}}
$$

This result is just what should be predicted from the fact that FAMA-NCS supports correct floor acquisition. The throughput in a fully connected network for FAMA-NCS has been shown to be [5]

$$
S=\frac{\delta}{\gamma^{\prime}+\delta+2 \tau+\frac{1}{\lambda}+e^{\tau \lambda}(\gamma+4 \tau)}
$$

Eqs. (15) and (14) indicate that, as the number of hidden terminals increases with respect to any given group, FAMA-NCS degrades to the case of a fully connected network in which the vulnerability period of an RTS becomes twice the length of the RTS, rather than the propagation delay. This is exactly the type of behavior of a packet-sensing FAMA protocol operating in a fully-connected network. Note that, because $\gamma<<\delta$, this behavior is far better than the degradation experienced by CSMA, in which the vulnerability period of a packet becomes twice its length, which is the behavior of the ALOHA channel.

\subsection{Performance with Hidden Terminals}

We compare FAMA-NCS and CSMA in networks with independent groups hidden from one another, and with one common central station. This type of experiment is similar to the ones used by Tobagi and Kleinrock [14].

We consider performance of the protocols in a wireless network with a bandwidth of $1 \mathrm{mb} / \mathrm{s}$. The size of the data packets is $4000 \mu \mathrm{s}$, the size of the RTS packet is $200 \mu \mathrm{s}$. The maximum one-way propagation delay across the channel is $6.4 \mu \mathrm{s}$ (a little over one mile).

Fig. 8 shows the throughput $(S)$ verses offered load $(G)$ of FAMA-NCS for different numbers of independent groups $(N)$.

Fig. 9 shows channel capacity (maximum attainable throughput) versus increasing numbers of independent groups $(N)$ for FAMANCS, non-persistent CSMA, and ALOHA. For the case of nonpersistent CSMA and ALOHA, we assume [8] that a separate perfect channel is used for acknowledgments to let a station know when its packet was received free of collisions, and that all acknowledgments are sent reliably. Therefore, the throughput of nonpersistent CSMA and ALOHA used for comparison with FAMA

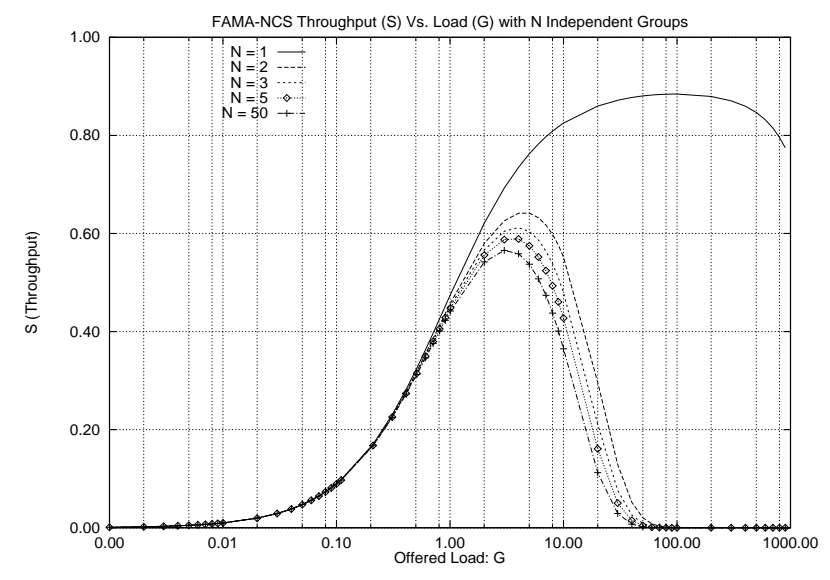

Figure 8: Throughput $(S)$ of FAMA-NCS verses offered load $(G)$

protocols is only an upper bound. Fig. 9 also shows a line for the capacity of FAMA-NPS in a fully connected network. The results indicate that FAMA-NCS's performance under hidden terminals becomes that of a packet-sensing FAMA protocol operating in a fully connected network. This is exactly the desired result. In contrast, as has been reported by Kleinrock and Tobagi, CSMA quickly degrades to ALOHA.

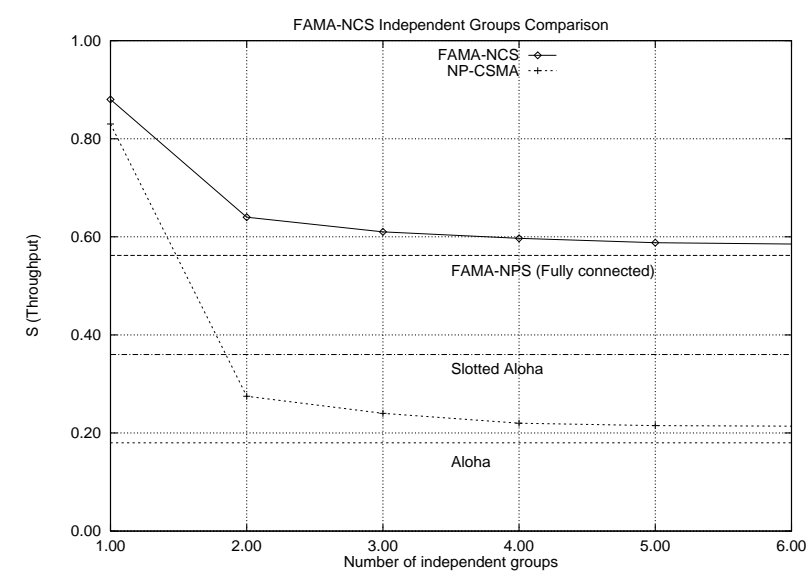

Figure 9: Throughput of FAMA protocols for increasing numbers of independent groups

We also looked at a complimentary-couple configuration [14]. In this configuration, a fraction of the population is hidden from the rest. We use two independent groups $(N=2)$ and vary the size of one group versus the other, such that $S_{1}=\alpha \cdot S$ and $S_{2}=(1-\alpha) \cdot S$. The total average arrival rate of RTSs is 
set to $G=5.0$, which corresponds to the arrival rate at which the maximum throughput is obtained when $\alpha=1 / 2$. Fig. 10 shows the channel capacity versus $\alpha$. This example illustrates that, while CSMA quickly degrades to ALOHA, the floor acquisition property of FAMA-NCS provides for a much smaller performance degradation.

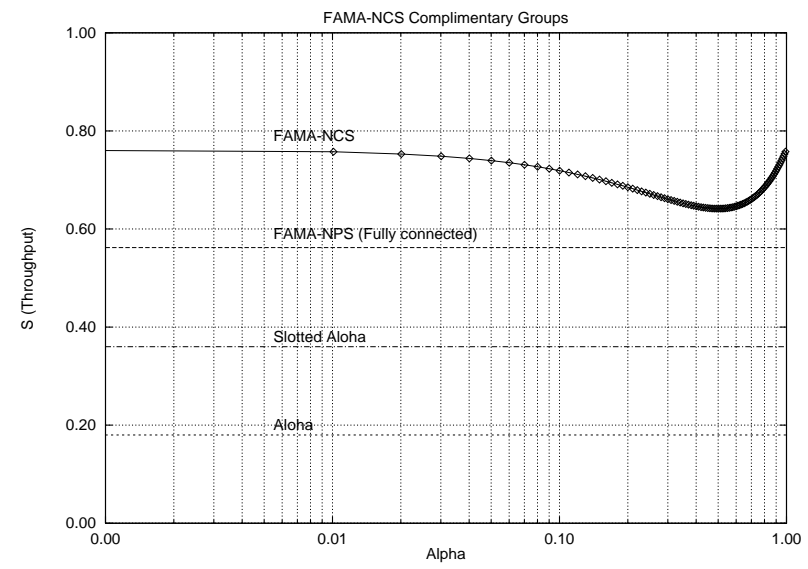

Figure 10: Throughput of FAMA-NCS in the complimentary couple configuration

\section{Simulation Results}

To validate our results on sufficient conditions for floor acquisition and the approximations made in our performance analysis, we carried out a number of simulations. The simulation runs the actual code used to implement the MAC protocols in embedded systems and, for the case of FAMA-NCS, this code is based on the specifications shown in Fig. 2.

We present results for the FAMA-NCS protocol using single packet transmissions as well as packet trains. Figure 11 shows the various configurations used by the simulations. Table 1 show the results for FAMA-NCS as compared to MACAW [1]. To illustrate the importance of carrier sensing, we chose to compare FAMANCS against MACAW instead of FAMA-NPS, because MACAW uses packet-sensing and RTS-CTS handshakes and its performance has been reported before by Bharghavan et al. [1]

For our simulation analysis we assumed single channel spread spectrum radios capable of transmitting at $256 \mathrm{Kbs}$. The stations are within four miles of each other, giving a maximum propagation delay of approximately 20 microseconds. The physical parameters of the radio assumed a null transmit-to-receive turnaround time and transmitter ramp-up time, we also assumed transmission preamble and framing of 0 bits. Our results are only meant for comparative purposes.

In configuration (a) of Fig. 11 all stations are within range of all others (no hidden terminals). Traffic was generated at each node (N1 - N6) directed to the base station. Configuration (b) has two groups of five nodes that can hear the nodes in their own group, but are hidden from nodes in the other group. Traffic is generated from each node in each group directed to the central base station, $B 1$. Configuration (c) has two base stations each with a group of five nodes sending traffic to it. The two groups cannot hear each other except for two nodes in each group that interfere with correspond-

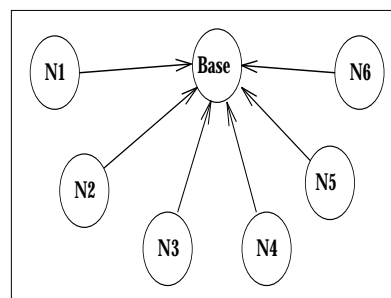

(a)

(c)

(d)
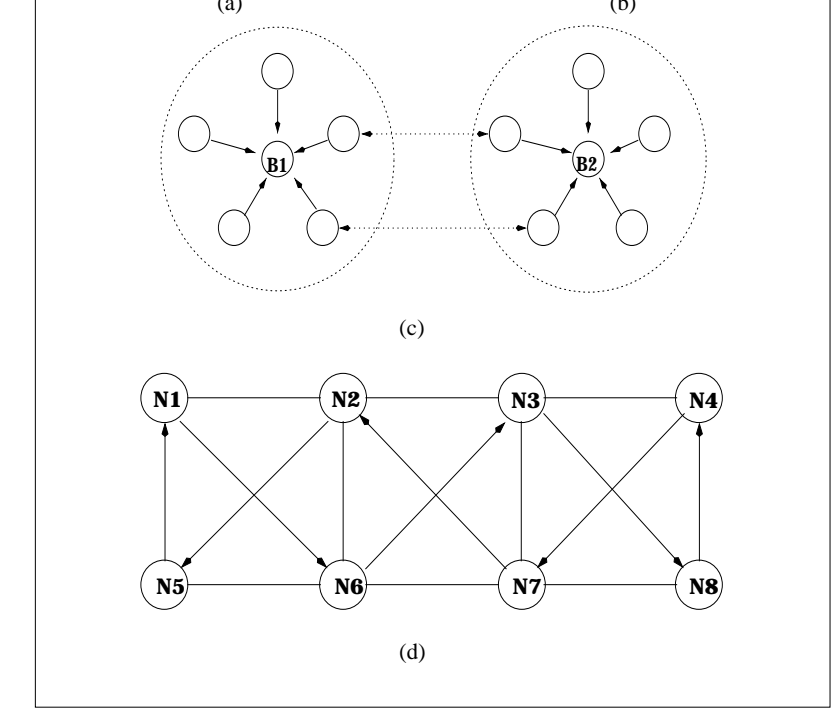

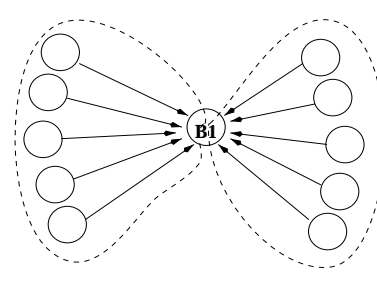

(b)
Figure 11: Simulation topologies used in testing FAMA protocols in hidden terminal environments

ing nodes in the other group (represented by the dashed arrows in the figure). Configuration (d) represents a multihop network of eight nodes. The lines between the nodes represent the radio connectivity of the network. The lines with arrows depict the flow of traffic from one node to another. Each node is generating a traffic stream to another node that at least three other nodes can hear, and is hidden from at least two of the other nodes in the network.

The traffic delivered to the nodes was sent at a constant rate with a packet size of 512 bytes on the channel (including all headers and framing). The maximum capacity of the channel at this bandwidth and packet size is approximately 63 packets per second. Table 1 reports the maximum throughput achieved by each of the protocols.

\begin{tabular}{||c|c|c|c||}
\hline Configuration & FAMA-NCS & FAMA-NCS train & MACAW \\
\hline \hline$(a)$ & .78 & .89 & .63 \\
\hline$(b)$ & .58 & .81 & .49 \\
\hline$(c) \mathrm{B} 1$ & .75 & .88 & .45 \\
\hline$(c) \mathrm{B} 2$ & .75 & .88 & .39 \\
\hline$(d)$ average & .49 & .67 & .06 \\
\hline$(d) \mathrm{N} 1,4,5,8$ & .57 & .81 & .07 \\
\hline$(d) \mathrm{N} 2,3,6,7$ & .42 & .54 & .05 \\
\hline
\end{tabular}

Table 1: Throughput results for various configurations

FAMA-NCS achieves a higher throughput than that of MACAW in all cases. For the case of a fully connected network (configuration (a)), FAMA-NCS attains a maximum throughput of $78 \%$, while MACAW achieves a $63 \%$ throughput. These results are as predicted 
by our approximate analysis of Section 4. For the case of MACAW, our simulation leads to a slotted behavior in which a slot lasts the duration of an RTS plus a maximum round-trip time. For the case of two independent groups competing for the same base station, FAMA-NCS has a maximum throughput of $58 \%$, while MACAW's achieves $49 \%$ maximum throughput. However, for the case of the two base stations with a small number of interfering nodes (configuration (c)), FAMA-NCS achieves a throughput of nearly twice that of MACAW, and in fact shows very little loss in overall throughput from interference due to hidden terminals $(78 \%$ without interference, $75 \%$ with interference).

In the multihop-network example (d) FAMA-NCS achieves an average throughput of $49 \%$, with the nodes on the corners (N1,N4,N5,N8) reaching 57\%, and the inside nodes reaching $42 \%$. This is somewhat lower than predicted by our analysis, but expected because the analysis assumes a base station that does not transmit data packets. In this network MACAW achieves a much lower throughput of $6 \%$ on the average, achieving $7 \%$ at the corner nodes, and $5 \%$ on the inside nodes.

As expected, FAMA-NCS with packet trains of up to five packets in a train improves over single-packet transmissions by about $14 \%$ in the fully connected network and $17 \%$ for the two-base station configuration. In the case of two independent groups sending to one central base station, the improvement is almost $40 \%$. For the multihop network FAMA-NCS packet trains provide an average throughput improvement of about $36 \%$.

The poor performance of MACAW (and FAMA-NPS for that matter) in a multihop network is a direct consequence of the fact that data packets can collide with other packets, i.e., that it cannot enforce "floor acquisition" in the presence of hidden terminals and emphasizes the benefits of using carrier sensing.

\section{Concluding Remarks}

We have analyzed the floor acquisition multiple access (FAMA) protocols for single-channel packet-radio networks with hidden terminals. FAMA protocols permit a station to acquire control of the channel dynamically before transmitting data packets. The floor acquisition strategy uses an RTS-CTS handshake and is based on two basic principles: (a) implementing a busy-tone mechanism using a single channel by making the receiver send CTSs that last long enough, or are repeated enough times, for the hidden senders to realize that they must back off; and (b) providing priority to those stations who successfully complete a handshake. The importance of our analytical work is illustrated by the fact that Theorem 2 predicts the poor performance of FAMA protocols using packet sensing when a node is subject to several hidden terminals. This negative effect of hidden terminals remained unnoticed even in detailed simulation experiments carried out for MACAW [1].

Although many MAC protocols have been introduced in the past based on RTS-CTS exchanges, we prove, for the first time, sufficient conditions under which an RTS-CTS dialogue becomes a floor acquisition strategy (i.e., one with which data packets are sent without ever colliding with other transmissions) with and without carrier sensing. Contrary to the conjectures made in prior work on MAC protocols based on collision avoidance [1,7], our verification and throughput analysis demonstrates that carrier sensing should be used in single channel networks because it substantially improves performance by enabling floor acquisition in the presence of hidden terminals.
We have shown through our analysis and supported by simulations that FAMA-NCS solves the hidden terminal problems of CSMA [14] in multihop networks because it is able to enforce floor acquisition. Our analysis illustrates the performance improvement obtained by allowing the transmission of packet trains in the clear, and a method to enable packet trains even with hidden terminals.

FAMA protocols have been demonstrated successfully in actual packet radios built with commercial off-the-shelf hardware and operating in multihop networks [18].

\section{References}

[1] V. Bharghavan, A. Demers, S. Shenker, and L. Zhang, "MACAW: A Media Access Protocol for Wireless LAN's," in Proc. ACM SIGCOMM '94, pp. 212-25, London, UK, Aug. 31-Sept. 2, 19941994.

[2] K. Biba, "A Hybrid Wireless MAC Protocol Supporting Asynchronous and Syncronous MSDU Delivery Services," Tech. Rep. Paper 802.11/91-92, IEEE 802.11 Working Group, 1992.

[3] K.-C. Chen, "Medium access control of wireless lans for mobile computing," IEEE Network, vol. 8, no. 5, pp. 50-63, 1994.

[4] A. Colvin, "CSMA with collision avoidance," Computer Commun., vol. 6, no. 5, pp. 227-35, 1983.

[5] C. L. Fullmer and J.J. Garcia-Luna-Aceves, "Floor Acquisition Multiple Access (FAMA) for Packet-Radio Networks," Proc. ACM SIGCOMM 95, Cambridge, MA, Aug. 28-Sept. 1, 1995.

[6] P802.11-Unapproved Draft: Wireless LAN Medium Access Control (MAC) and Physical Specifications, IEEE, January 1996.

[7] P. Karn, "MACA - a new channel access method for packet radio," in ARRL/CRRL Amateur Radio 9th Computer Networking Conference, pp. 134-40, ARRL, 1990.

[8] L. Kleinrock and F. A. Tobagi, "Packet switching in radio channels: Part I - carrier sense multiple-access modes and their throughputdelay characteristics," IEEE Trans. Commun., vol. COM-23, no. 12, pp. 1400-1416, 1975 .

[9] B. M. Leiner, D. L. Nielson, and F. A. Tobagi, eds., Proceedings of the IEEE, vol. 75, IEEE, January 1987.

[10] W. F. Lo and H. T. Mouftah, "Carrier Sense Multiple Access with Collision Detection for Radio Channels," in IEEE 13th Int'l Commun. and Energy Conf., pp. 244-47, IEEE, 1984.

[11] R. Rom, "Collision Detection in Radio Channels," in Local area and multiple access networks, pp. 235-49, Computer Science Press, 1986.

[12] G. S. Sidhu, R. F. Andrews, and A. B. Oppenheimer, Inside AppleTalk, Second Edition. Addison-Wesley Publishing Company, Inc., 1990.

[13] H. Takagi and L. Kleinrock, "Output processes in contention packet broadcasting systems," IEEE Trans. Commun., vol. COM-33, no. 11, pp. 1191-9, 1985.

[14] F. A. Tobagi and L. Kleinrock, "Packet switching in radio channels: Part II - the hidden terminal problem in carrier sense multiple-access modes and the busy-tone solution," IEEE Trans. Commun., vol. COM23, no. 12, pp. 1417-1433, 1975.

[15] F. A. Tobagi and L. Kleinrock, "The Effect of Acknowledgment Traffic on the Capacity of Packet-Switched Radio Channels," IEEE Trans. Commun., vol. COM-26, no. 6, pp. 815-826, 1978.

[16] B. Vaduvur, Access, Addressing and Security in Wireless Packet Net works. PhD thesis, University of California, Berkeley, Computer Science Department, 1995.

[17] C. Wu and V. O. K. Li, "Receiver-initiated busy-tone multiple access in packet radio networks," ACM SIGCOMM 87 Workshop: Frontiers in Computer Communications Technology, Stowe, VT, USA, 11-13 Aug. 1987.

[18] "WINGS for Internets," www.cse.ucsc.edu/research/ccrg/projects/wings.html 